\title{
The Young Judokas Cardiorespiratory System Reactivity on the Effect of Muscle Loads
}

\author{
Pseunok A.A. ${ }^{1, *}$ Mugotlev M.A. ${ }^{1}$ Silantiev M.N. ${ }^{1}$ Hasanova R.A. ${ }^{2}$ \\ ${ }^{1}$ Adygeya State University, Maykop, Russia \\ ${ }^{2}$ Kuban State Medical University, Krasnodar, Russia \\ *Corresponding author. Email: pseunokk@mail.ru
}

\begin{abstract}
The study was aimed to assess the reactivity of the cardiorespiratory system of young judokas of 12-14 years old, depending on age, health condition and sports specialization. In the longitudinal mode for two years, 32 young athletes were examined. The study was conducted using methods of analyzing heart rate, determining the function of external respiration and the electrolyte composition of saliva. Training loads have a beneficial effect on the body of young judokas, this is expressed in strengthening parasympathetic and stabilizing sympathetic influences in the regulation of heart rhythm in a state of relative rest. This nature of changes in the indicators of the cardiorespiratory system and the electrolyte content was evaluated as the economization of the activity of the heart at rest and the availability of capabilities for performing training loads. Regular physical exercises of young judokas cause positive stimulation of all functional systems, primarily the reactivity of the cardiorespiratory system. The lack of centralization of function control was revealed, which indicates the balance of the autonomous regulation system (sympathetic and parasympathetic divisions of the autonomic nervous system) by the profitability principle. High energy and adaptive capabilities of young judokas indicate the optimal state of regulatory systems. The reactivity of the cardiorespiratory system depends on age, training endurance loads and the individual characteristics of the child's body. In young judokas of 12-14 years old, the leading adaptive-compensatory reaction is an increase in the depth of breathing. The vegetative support system is being improved. The results scientifically substantiate preventive and recreational measures aimed at normalizing the functions of the body.
\end{abstract}

Keywords: Adaptation, heart rate, saliva electrolytes, cardiorespiratory system, young judokas, muscle loads

\section{INTRODUCTION}

Motor activity is one of the factors determining the structural and functional formation of the cardiorespiratory system. The study of the cardiorespiratory system under various motor conditions evaluates the functional and reserve capabilities of the body. In this regard, the study of the cardiorespiratory system acquires important theoretical significance for medicine and physiology.

Studies of the cardiorespiratory system in athletes who are systematically exposed to physical activity of different volumes and intensities are important for the formation of sustainable knowledge regarding the basic physiological adaptation of the body to muscle work.

Physiological indicators, reflecting, to one extent or another, the state of the mechanisms of autonomic regulation of cardiac activity, reliable markers for assessing the physiological state and predicting physical fitness, control of a training session can be used $[2,8]$.

\section{PROBLEM STATEMENT}

The study of the heart rhythm structure is crucial in age physiology, for determining the role of regulatory mechanisms of the functioning of the cardiovascular system in the formation of general and special training $[1,11,12]$. In addition, the reliability of the functioning of the body largely depends on the state of balance of electrolytes and their concentration in biological fluids [5].

Sports is accompanied by adaptive changes in the oxygen supply of the body due to the activation of the hemodynamic functional reserves [3]. However, the features of motor regime influence on the cardiorespiratory system of young judokas is not fully studied.

The ability to do sports mainly depends on individual characteristics - physiological reactivity and the effectiveness of urgent adaptation mechanisms. Adaptation changes depend on genetic factors determining a predisposition to muscle activity of various intensities and durations $[7,13]$.

The functional system of external respiration providing the necessary volume of pulmonary ventilation in each respiratory cycle, is part of a more complex functional system that supports the optimal respiratory rate of the body for metabolism. The volume of pulmonary ventilation at any given time is the result of a complex interaction of humoral and nerve excitations that characterize respiratory need [9].

An important indicator reflecting oxygen consumption, the functional state of the cardiorespiratory system. There 
is a relationship between circulation and respiration factors that affect the amount of oxygen consumed. During exercise, oxygen consumption increases significantly. This places high demands on the function of the cardiovascular and respiratory systems. Therefore, the cardiorespiratory system during muscular work changes depending on the intensity of physical activity. Motor activity, of course, has a positive effect on the child's body, but significant loads performed during training and competitions to achieve a sports result can adversely affect children's health. Too early sports specialization may harm health and physical development of a young athlete. The right time to start certain training should be determined considering the ontogenetic development [6].

Though there are no longitudinal studies of the cardiorespiratory system, which does not fully characterize the adaptive capabilities of the body of young athletes at different stages of ontogenesis depending on experience and sports specialization.

High sports results is achieved by great physical exertion, significant psycho-emotional stress in a competitive environment and other stressful effects. These factors require more subtle and clear diagnostic methods. In this regard, there is a great attention paid to the methods reflecting not only the state of individual organism and systems, but also determine the level of regulation of various physiological functions. A double burden is noticed when playing sports, there occurs continuous growth processes [14]. This happens long with adaptation to great physical and psycho-emotional stresses associated with the training process and any physical activity.

\section{RESEARCH QUESTIONS}

The research question is to study the adaptive rearrangements of management structures and the functioning of the cardiorespiratory system of young athletes according to their age, training experience and the effects of physical loads.

\section{PURPOSE OF THE STUDY}

To evaluate the reactivity of the cardiorespiratory system of young judokas depending on age, fitness and sports specialization.

\section{RESEARCH METHODS}

The study of heart rate was conducted using the method of R.M. Baevsky, experiment of a young athlete after 5 minutes in a sitting position and after exercise with the help of the Polyspectrum-Rhythm electrocardiograph. A number of parameters were determined, such as: mode (Mo), mode amplitude (AMo), variation range $(\Delta \mathrm{X})$, stress index (IN), autonomic equilibrium index (IIR), vegetative equilibrium index (PVR).
The measured vital capacity of the lungs, tidal volume and reserve volume of inspiration were measured to study the function of external respiration. The study was conducted using the Spiro-Spectrum instrument of the NeuroSoft company, a computer, and the corresponding software.

The determination of electrolytes in saliva was carried out by the photocolorimetric method using the KFK-3 device. Sodium was determined by the microcolorimetric method. The potassium content in saliva was determined by a photocolorimetric micromethod.

The research results were processed using the method of variation statistics with the calculation of the arithmetic mean $(\mathrm{M})$, arithmetic mean error $(\mathrm{m})$, student's confidence criterion $(\mathrm{t})$ and probability level $(\mathrm{P})$.

\section{FINDINGS}

The results of the study showed that changes in heart rate are wave-like, increasing slightly after exercise, with a low amplitude of oscillations (P <0.05). Sympathetic influences are amplified by the second training macrocycle regarded as the norm [4].

The vegetative rhythm indicator during the first year varies slightly, but at the end of the second year it decreases significantly. It is assumed that the load is satisfied due to the autonomous regulation loop without the participation of cortical-limbic structures. Two-year observations of heart rate dynamics revealed one peak when the voltage index values significantly increased $(\mathrm{P}$ <0.05) (Fig. 01, 02). It depends on various factors: chronobiology, emotional stress caused by competitions, in which most of the subjects took an active part. The study revealed low activation of central regulatory mechanisms in the non-competitive period considering as a favorable factor.

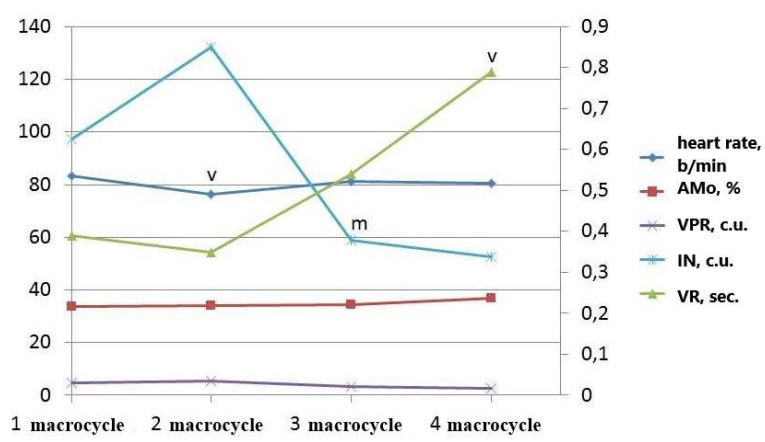

Figure 1 The dynamics of the heart rate of young athletes 12-14 years old, practicing judo, alone. Significance of differences between indicators $(t)$ : $\mathrm{m}-$ the first and second years of the study; $\mathrm{v}-$ during the year. 


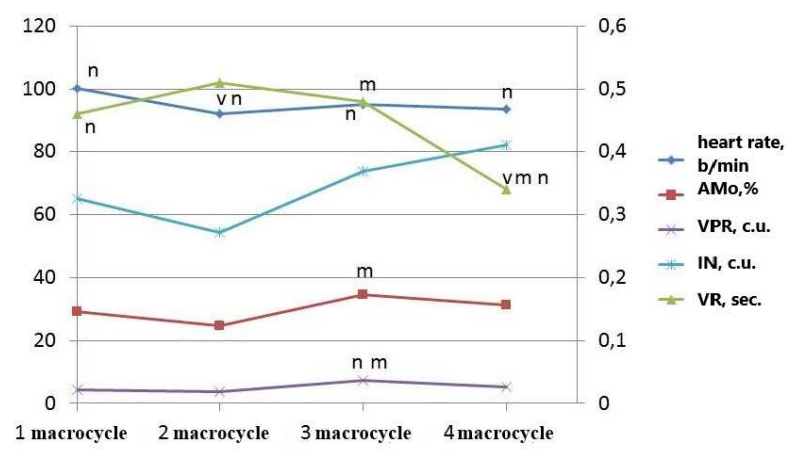

Figure 2 The dynamics of the heart rate of young athletes 12-14 years of age, engaged in judo after exercise. Significance of differences between indicators $(\mathrm{t}): \mathrm{n}-$ at rest and after exercise; $\mathrm{m}$ - the first and second years of the study; $v$ - during the year.

Thus, regular physical activities performed by young judokas cause positive stimulation of all functional systems, primarily the reactivity of the cardiorespiratory system.

The obtained results convincingly indicate the lack of function control centralization, the balance of autonomous regulation systems (sympathetic and parasympathetic divisions of the autonomic nervous system) by the principle of profitability. High energy and adaptive capabilities of the body of young judokas indicate the optimal state of regulatory systems.

The oxygen transport system of the blood is developed in the period of 12-14 years, due to the increase in the size and volume of the lungs. There occurs a gradual increase in the efficiency of oxygen supply to the body. However, in this period the bronchial resistance to the air flow, relatively shallow respiration, the large expenditure of energy on the activity of the respiratory muscles are still not large enough, with intensive pulmonary ventilation, the use of oxygen in the lungs is inefficient.

A study of the respiratory system indices of young judokas of 12-14 years old showed that the lung capacity increases by the end of the fourth macrocycle $(\mathrm{P}<0.05)$. It should be noted that this occurs at different time stages due to its various components.

So, if during the first three macrocycles the increase in $\mathrm{VC}$ is due to the tidal volume, then by the fourth macrocycle it decreases $(\mathrm{P}<0.05)$, while the reserve volume of inspiration increases immensely. According to these changes in the parameters of the respiratory system suggests, by the end of the second training macrocycle, the depth of breathing increases. An increase in the depth of breathing due to an increase in tidal volume is one of the adaptation-compensatory mechanisms for providing oxygen homeostasis. By the end of the fourth macrocycle, there is an increase in the efficiency of the functioning of the respiratory system. It depends on the fact that respiration becomes superficial, but has large functional reserves (Fig. 03).

Thus, young judoka of this age experiences an increase in the working level of vegetative functions.

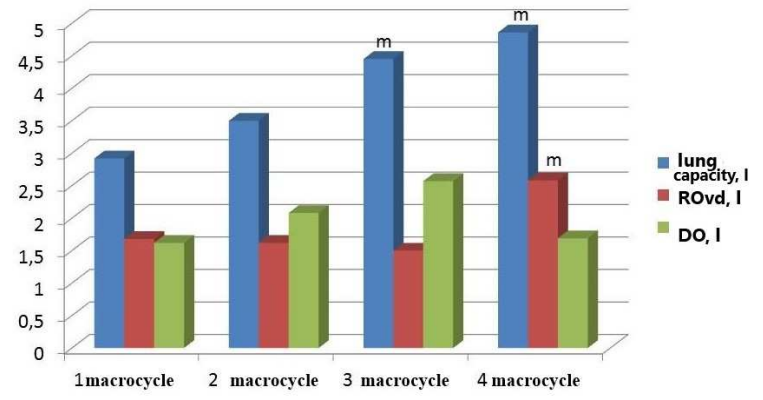

Figure 3 The dynamics of the respiratory system of 1214 years old judokas. Significance of differences between indicators $(\mathrm{t}): \mathrm{m}-$ the first and second years of the study

The results of the study of the electrolyte composition of young judokas saliva indicate a decrease in the amount of sodium and potassium by the end of the fourth macrocycle ( $\mathrm{P}<0.05)$ (Fig. 04).

Such dynamics is determined by a decrease in potassium excretion and an increased reactivity of sodium regulatory mechanisms, since the $\mathrm{Na}+/ \mathrm{K}+$ ratio is largely determined by aldosterone, angiotensin II, and natriuretic peptides. Atria synthesize a vasodilator and diuretic substance - atrial natriuretic peptide. This peptide is placed and effuses from secretory vesicles localized in atrial cardiomyocytes, as well as in sinoatrial cells. It is believed that atrial natriuretic peptide reduces the activity of the sympathetic nervous system and potentiates cardiac parasympathetic effects. According to the mechanism of action, it is known as an antagonist of 1-adrenergic receptors. The peptide is characterized by a direct negative chronotropic effect, which explains the tendency to a decrease in heart rate [3].

The nature of the change in the $\mathrm{Na}+/ \mathrm{K}+$ coefficient indicates insignificant changes in the ratio of sodium and potassium in saliva. It does not cause significant changes in the proportion of non-specific adaptive reactions in response to the training load.

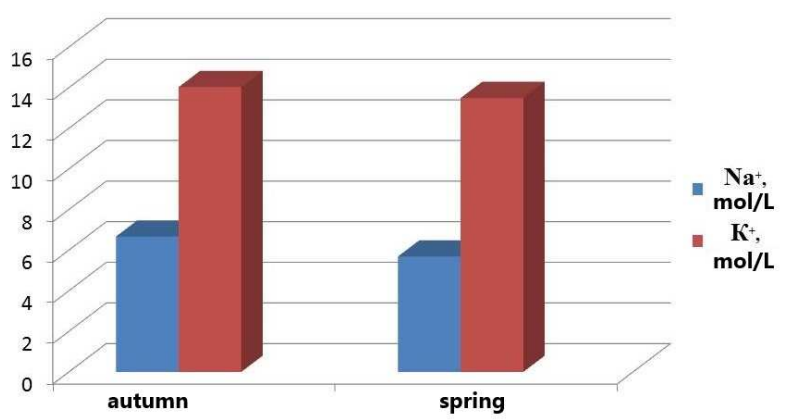

Figure 4 The dynamics of electrolytes concentration in the saliva of 12-14 years old young judokas

\section{CONCLUSION}

The effect of physical training on the child's body, particularly, on the cardiorespiratory system is not a 
surprise. This studies, according to most researchers, increase the level of functional state and nonspecific resistance of the body. This provides a more effective adaptation of the body to environmental conditions including training and physical loads.

Training loads have a beneficial effect on the body of young judokas: strengthening parasympathetic and stabilizing sympathetic influences in the regulation of heart rhythm in a state of relative rest. This nature of changes in the indicators of the cardiorespiratory system and the electrolyte content was regarded by us as economization of the activity of the heart at rest and the availability of reserves for performing training loads.

Thus, the study revealed the characteristics of the cardiorespiratory system reactivity depending on age, training loads and individual characteristics. 12-14 years old young judokas have developed adaptive-compensatory reaction and improved vegetative.

\section{REFERENCES}

[1] R.M. Bayevsky, A.P. Berseneva, Adaptation possibilities and the concept of physiological norm, Thes. doc. XVIII Congress of the Physiological Society I.P. Pavlova, GEOTAR-MED, Kazan, Moscow, 2001, 304 p.

[2] R.M. Baevsky, A.G. Chernikova, Assessment of adaptive risk in the system of individual prenosological control, Russ. Physiol. J. THEM Sechenova 100(10) (2014) 1180-1194, Moscow.

[3] Yu.S. Vanyushin, R.R. Khayrullin, D.E. Elistratov, The value of the coefficient for a comprehensive assessment of the cardiorespiratory system for diagnosing the functional state of athletes, Theory and Pract. of Phys. Culture 5 (2017) 59-61.

[4] A.R. Galeev, L.N. Igisheva, E.M. Kazin, Heart rate variability in healthy children aged 6-16 years, Human Physiol. 28(4) (2002) 54-58.

[5] A.V. Kutina, A.A. Kuznetsova, Yu.V. Natochin, Cations in Human Serum, Uspekhi Fiziologicheskikh Nauk 36(3) (2005) 3-32.
[6] G.A. Makarova, Sports Medicine, Soviet Sport, Moscow, 2002, 480 p.

[7] I.B. Nazarov, Essentially-substantive substantiation of the reflective component of a person's sports culture, Physiological and Biochemical Foundations and Pedagogical Technologies for Adaptation to Different Physical Exercises, All-Russian scientificpractical conference with international participation, dedicated to the memory of Dr. Biol. sciences, professors A.S. Chinkina, Kazan, 27-28 November 2014, pp. 201-204.

[8] T.V. Solomina, L.I. Vovchenko, Planning the training process of swimmers based on continuous monitoring of the heart rhythm, Theory and Pract. of Phys. Culture 4 (2000) 28-30.

[9] K.V. Sudakov, Functional systems, Monograph, Publ. house of RAMS, Moscow, 2011, 320 p.

[10] V.N. Khirmanov, Natriuretic peptides - a family of circulatory system regulators, Cardiol. and Cardiovascular Surgery 10(1) (2017) 3-25.

[11] R. Bailona, Analysis of heart rate variability during exercise stress testing using respiratory information, Biomed. Signal Processing and Control 5 (2010) 299-310.

[12] N.V. Mishchenko, T.A. Trifonova, I.A. Klimov, Express assessment of the students' adaptive abilities and revealing of the risk group, Asian Social Sci. 11 (2015) 313-320.

[13] P. Nickel, F. Nachreiner, Sensitivity and Diagnostics of the $0.1-\mathrm{Hz}$ Component of Heart Rate Variability as an Indicator of Mental Workload, Human Factors 45(4) (2003) 575-590.

[14] A.A. Pseunok, M.A. Mugotlev, M.N. Silantiev, Features of heart rate and electrolyte composition of saliva of young judoists 10-12 years, Theory and pract. of phys. Culture 12 (2018) 48-51.

[15] J. Sztajzel, Heart rate variability: a noninvasive electrocardiographic method to measure the avtonomic nervous system, Swiss med wkly 143 (2004) 514-522. 